\title{
CIS
}

\section{OPTICAL ADD DROP MULTIPLEXERS (OADMS) IN UW-WDM OPTICAL PASSIVE COMMUNICATION NETWORKS}

\author{
Abd El-Naser A. Mohammed, Ahmed Nabih Zaki Rashed, Mahmoud M. A. Eid \\ Electronics and Electrical Communication Engineering Department \\ Faculty of Electronic Engineering, Menouf 32951, Menoufia University, Egypt \\ e-mail: ahmed_733@yahoo.com
}

\begin{abstract}
In the present paper, we have been modeled numerically and parametrically the high and best performance functions of optical add drop multiplexers (OADMs) for ultra wide wavelength division multiplexing technique with ultra wide space division multiplexing technique in advanced optical communication networks and photonic networking over the assumed set of parameters. Moreover, we have analyzed and investigated the maximum time division multiplexing (MTDM) and soliton transmission techniques to be processed to handle bit rate either per link or per channel for cables of multi-links (20-120 links/core). Where maximum number of transmitted channels in the range of 1000-4800 channels are processed to handle the product of bit rate either per channel or per link for cables of multilinks of silica cable core fabrication material. The MTDM or soliton transmission bit rates either per link or per channel are also treated over wide range of the affecting parameters under the ambient temperature variations. Also, the performance characteristics of the OADMs are taken as the major interest in optical networks to handle maximum transmission bit rates for the supported subscribers.
\end{abstract}

Keywords: Optical add drop multiplexers, Advanced optical communication networks, Ultra-wide wavelength division multiplexing (UW-WDM), Ultra-wide space division multiplexing (UW-SDM).

\section{INTRODUCTION}

The introduction of optical add drop multiplexers into optical networks allows traffic to be inserted, removed and, most importantly, bypassed. Additionally, functions such as protection, drop/continue, loop-back and wavelength reuse of the optical channels can be supported by the OADMs. Wavelength reuse means that the dropped channel does not pass through to the next OADMs [1]. Instead a new channel of the same wavelength can be added. Drop and continue means that the channel is both dropped at the node but also allowed to pass through to the next OADMs [2]. Depending on, which network the OADMs should be used in, different requirements are set, based on cost, capacity, redundancy and flexibility. OADMs can be realised in various technologies [3]. From a transmission point of view OADMs can be classified into notching and demultiplexing. The DMUX based solution separates all the incoming wavelengths and then combines them again after dropping and adding wavelengths. The notch type only separates the wavelength(s) to be dropped. Because the notch type doesn't affect bypassed channels, the cascaded passband and crosstalk performance can be improved compared to the demultiplexing OADM. The crosstalk component at the OADM output port originates from their poor suppression of the drop channel (assumed wavelength reuse), which leads to interferometric crosstalk [4]. At the drop port, crosstalk comes from low suppression of the other channels. Similar to the optical terminal multiplexer (OTM), the OADMs can be divided into a single port with static wavelength assignment, a single port with dynamic wavelength assignment and a multi port with static and dynamic wavelength assignment. The single port with static wavelength assignment is mainly used in hubbed structures, where the OADMs are connected to a central hub, e.g. in the metropolitan network. In order to utilize network resources in a more efficient way, the OADMs with dynamic wavelength assignment are preferred when traffic variations are comparable to network capacity [5]. The multi port OADMs can be utilized when the network is characterized by a uniform traffic distribution and high capacity. The optical add-drop multiplexer is one of the key components for dense wavelength division multiplexing (DWDM) networks. The optical add/drop multiplexer (OADM) is a key component for wavelength division multiplexing (WDM) systems. Many types 
of OADMs have been demonstrated based on different optical devices. These devices include arrayed-waveguide gratings (AWG) [6], MachZehnder interferometers (MZI) with fiber Bragg gratings (FBG), and optical circulators with FBGs. Although AWGs have better potential for integration and appear to be more robust and tolerant to fabrication processes, they offer much less flexibility than FBGs as regards the shaping of their spectral response, and therefore can lead to worst performances when used as optical filters in some WDM systems [7]. OADM consists of three main subsystem, a wavelength selective demultiplexer, a switching subsystem and a wavelength multiplexer. Each OADM is expected to handle at least two distinct wavelength channels each with a coarse granularity of $2.5 \mathrm{Gbit} / \mathrm{sec}$ or higher (signals with finer granularities are handled by logical switch node such as SDH/SONET digital cross connects or ATM switches). There are eight ports for add and drop functions, which are controlled by four lines of optical switches The functions of OADM include node termination, drop and add, routing, multiplexing, providing mechanism of restoration for point-to point, ring and mesh metropolitan and also customer access network in fiber to the home (FTTH). By setting up the optical switch configuration, the device can be programmed to function as other optical devices such as multiplexer, demultiplexer, coupler, wavelength converter (with fiber grating filter configuration), OADM, wavelength round about and etc for a single application. The enormous grow in the demand of bandwidth is pushing the utilization of fiber infrastructures to their limits [8]. To fulfill this requirement the constant technology evolution is substituting the actual single wavelength systems connected in a point-to-point topology by UWWDM) systems, creating the foundations for the optical transport network (OTN). OADMs are the simplest elements to introduce wavelength management capabilities by enabling the selective add and drop of optical channels. UW-WDM optical access network with OADM may provide a high reliable, low cost effective and scalable optical access network, since the OADM is based on lowloss, low-cost passive optical devices and does not need any power supply [9]. In the present work, we have presented and developed the OADMs to be used in ultra wide wavelength division multiplexing with ultra wide space division multiplexing in advanced optical communication networks, which are capable of dealing with thousand to multi several thousand channels arbitrarily selected. The OADMs are used for selectively dropping and inserting optical signals into a transparent UW-WDM optical communication networks. The OADMs into optical communication networks allow traffic to be inserted, removed and, most importantly, bypassed. Additionally, functions such as protection, drop/continue, loop-back and wavelength reuse of the optical channels can be supported by the OADMs. The investigation of propagation technique is processed to handle both bit rates either per link or per channel and maximum number of transmitted channels in the range of 1000-4800 for cables of multi-links (20-120 links/core). OADMs allow customers to optimize the use of existing fiber by adding or dropping channels on a per-site basis, thereby maximizing fiber bandwidth.

\section{SCHEMATIC FUNCTION VIEW OF OPTICAL ADD DROP MULTIPLEXER (OADM)}

Any subscriber loop architecture for wideband applications should have certain desirable properties. The access equipments in subscriber premises should be low cost and easy to maintain. The high cost equipments should be in central office. This reduces the cost per subscriber for the network. The network should provide large bandwidth to the subscribers. Further, as all subscribers will not initially subscribe to high bandwidth services, the architecture should be able to provide low bandwidth services at much cheaper price. Later when the subscriber would like to upgrade to a high bandwidth service, the service provider should be able to do so at a small additional cost.

Optical add drop multiplexer can be made using acoustic-optic or interferometric effect. In an add drop multiplexer there are three ports: input port, add-drop port, and output port. Assuming that there are $\lambda_{\mathrm{i}} \ldots . \lambda_{\mathrm{n}-1}, \lambda_{\mathrm{n}}$ wavelength at the input and $\lambda_{\mathrm{i}}$ is dropped, then at add-drop port $\lambda_{i}$ is diverted. At the output port all the other wavelengths $\left(\lambda_{1} \ldots \lambda_{\mathrm{i}-}\right.$ $\left.{ }_{1} \lambda_{i+1} \ldots \lambda_{n-1} \lambda_{n}\right)$ will be as in Fig. 1 . When wavelength $\lambda_{\mathrm{i}}$ is added then at the input port $\lambda_{\mathrm{i}}$ should not be there (i.e., input has $\left.\lambda_{1} \ldots \lambda_{\mathrm{i}-1} \lambda_{\mathrm{i}+1} \ldots \lambda_{\mathrm{n}-1} \lambda_{\mathrm{n}}\right)$. At the output port all the wavelengths $\left(\lambda_{1} \ldots \lambda_{\mathrm{n}-1} \lambda_{\mathrm{n}}\right)$ will be as in Fig. 2.

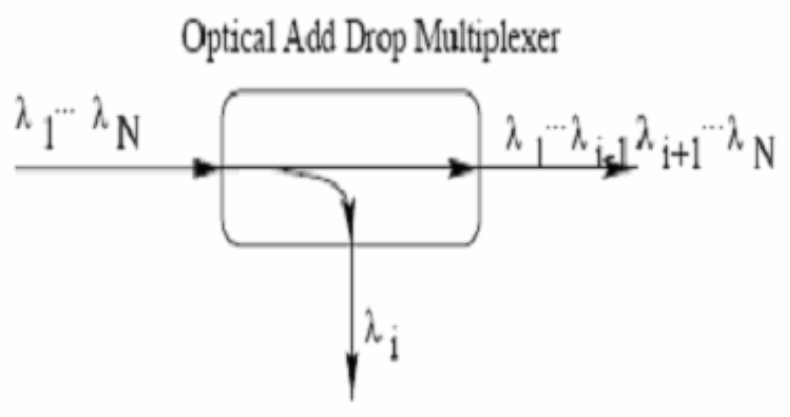

Fig. 1 - Drop function in OADM 


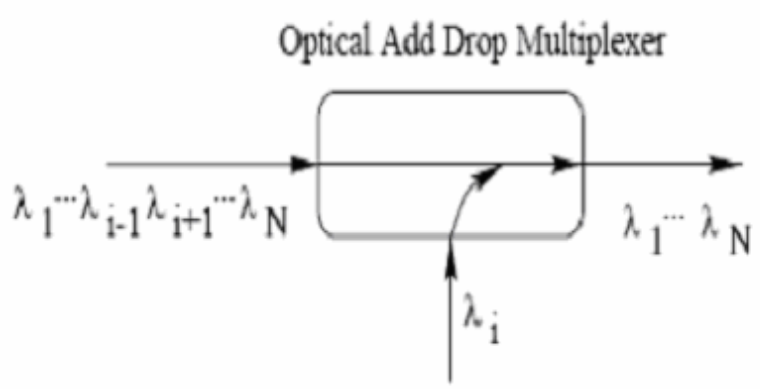

Fig. 2 - Add function in OADM

\section{BASIC MODEL AND EQUATIONS ANALYSIS}

We investigate the basic MTDM transmission technique to transmit 1000-4800 channels based on ultra wide wavelength division multiplexing (UWWDM), in the interval of 1.45 up to $1.65 \mu \mathrm{m}$ wavelengths. For the reality from the points of view of the spectral dependences of the different fiber characteristics [10], we employ also the ultra wide space division multiplexing (UW-SDM) where 1000-4800 channels are divided into subgroups each subgroup has its own spectral characteristics as in Fig. 3. With total number of links, $N_{L}=\{20,21$, $22, \ldots . \quad 50, \quad 51, \quad 52, \quad \ldots \quad 90, \quad 91$, $92, \ldots \ldots \ldots \ldots \ldots \ldots 120\}$ Links. With $\mathrm{JS}=\{1,2,3$, $\left.4,5, \ldots \ldots \ldots \ldots \ldots \ldots \mathrm{N}_{\mathrm{L}}\right\}$.

$$
\text { where: } \begin{aligned}
& \Delta \lambda_{L}=\Delta \lambda / N_{L} \equiv \text { Link spacing } \\
& \delta \lambda_{s}=\Delta \lambda /\left(N_{c h} \cdot N_{L}\right)=\Delta \lambda_{L} / N_{c h} \\
& \delta f \mid=\delta \lambda / \lambda_{\text {ave }}^{2} \cdot C \\
&|\Delta f|=\left\{\Delta \lambda_{L} / N_{c h} \cdot \lambda_{\text {ave }}^{2}\right\} . C
\end{aligned}
$$

where: speed of light in the optical fiber cable medium, $\mathrm{C}=3 \times 10^{8} \mathrm{~m} / \mathrm{sec}, \quad \mathrm{N}_{\mathrm{ch}} \equiv$ Number of channels/optical link, $\mathrm{N}_{\mathrm{L}} \equiv$ Total number of optical links/fiber cable core, and $\Delta \lambda=\lambda_{\mathrm{f}}-\lambda_{\mathrm{i}}=1.65-1.45$ $=0.2 \mu \mathrm{m}$.

$$
\lambda_{s}(\text { initial }) / \text { link }=1.45+(J S-1) \delta \lambda_{s}
$$

where the suffix " $f$ " denotes the final value and " $i$ " denotes the initial value, $\lambda_{\text {ave }}$ is the average

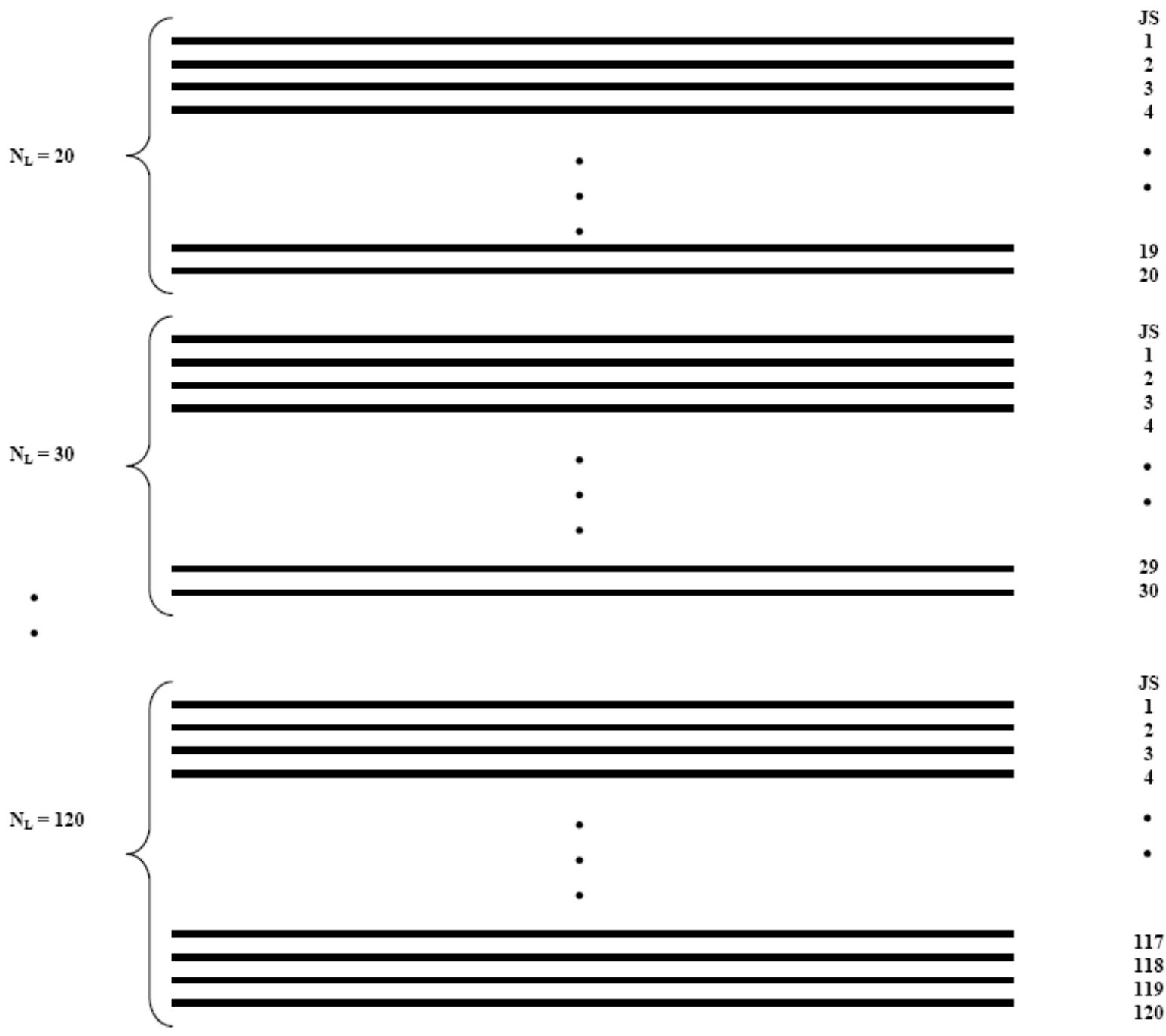

Fig. 3 - Optical link map through fiber cable core 
wavelength over the link of order JS, JS is the order of the link where $1 \leq \mathrm{JS} \leq \mathrm{N}_{\mathrm{L}}, \mathrm{N}_{\mathrm{L}}$ is the total number of links, $\lambda_{\mathrm{si}}$ is the initial wavelength at the link JS, and $\lambda_{\mathrm{sf}}$ is the final wavelength at the link JS. Due to the nonlinear limitations [11], so that the signal power $\mathrm{P}_{\mathrm{so}}$ must satisfies the inequality: i.e.,

$$
P_{\text {so }} \delta f \leq 500 / N_{c h}^{2} \text { watt. GHz }
$$

Also, the optical signal wavelength span $1.45 \leq \lambda$, $\mu \mathrm{m} \leq 1.65$ is divided into intervals equal to:

$$
\Delta \lambda_{0}=0.2 / N_{L}, \mu \mathrm{m} / \text { Link. }
$$

The average optical signal wavelength $\lambda_{\text {ave }}$ over a link of order JS can be estimated by:

$$
\lambda_{\text {ave }}=0.5 \Delta \lambda_{0}(J S+1)
$$

\section{A. SILICA CABLE CORE MATERIAL}

The refractive-index of the fabrication material of fiber cable core is given by the following [12]:

$$
n^{2}=1+\frac{B_{1} \lambda^{2}}{\lambda^{2}-B_{2}^{2}}+\frac{B_{3} \lambda^{2}}{\lambda^{2}-B_{4}^{2}}+\frac{B_{5} \lambda^{2}}{\lambda^{2}-B_{6}^{2}}
$$

The parameters of Sellmeier equation coefficients for silica cable core material as a function of temperature as follows: $\mathrm{B}_{1}=10.6684293, \mathrm{~B}_{2}=$ $0.030156485 .\left(\mathrm{T} / \mathrm{T}_{0}\right)^{2}, \mathrm{~B}_{3}=3.043224218 \times 10^{-3}, \mathrm{~B}_{4}=$ $1.1134751235 .\left(\mathrm{T} / \mathrm{T}_{0}\right)^{2}, \mathrm{~B}_{5}=1.54133408, \mathrm{~B}_{6}=1.104$ $\mathrm{x} 10^{3}$. Where $\mathrm{T}$ is the temperature of the material, ${ }^{\circ} \mathrm{C}$, and $\mathrm{T}_{0}$ is the reference room temperature and is considered as $25^{\circ} \mathrm{C}$. The first differentiation of Eq. (9) w. r. t $\lambda$ yields:

$$
\frac{d n}{d \lambda}=-\frac{1}{n}\left[\frac{B_{1} \lambda^{2}}{\left(\lambda^{2}-B_{2}^{2}\right)^{2}}+\frac{B_{3} \lambda^{2}}{\left(\lambda^{2}-B_{4}^{2}\right)^{2}}+\frac{B_{5} \lambda^{2}}{\left(\lambda^{2}-B_{6}^{2}\right)^{2}}\right],
$$

Then the second and third differentiation of Eq. (9) w. r. $t \lambda$ yields:

$$
\begin{gathered}
\frac{d^{2} n}{d \lambda^{2}}=\frac{1}{n}\left[\begin{array}{l}
\frac{B_{1}\left(\lambda^{2}-B_{2}^{2}\right)-4 \lambda^{2}}{\left(\lambda^{2}-B_{2}^{2}\right)^{3}}+\frac{B_{3}\left(\lambda^{2}-B_{4}^{2}\right)-4 \lambda^{2}}{\left(\lambda^{2}-B_{4}^{2}\right)^{3}} \\
+\frac{B_{5}\left(\lambda^{2}-B_{6}^{2}\right)-4 \lambda^{2}}{\left(\lambda^{2}-B_{6}^{2}\right)^{3}}
\end{array}\right], \\
\frac{d^{3} n}{d \lambda^{3}}=-\frac{1}{n}\left[\begin{array}{l}
\frac{12 B_{1} \lambda^{2}\left(\lambda^{3}+B_{1}^{2} \lambda\right)}{\left(\lambda^{2}-B_{2}^{2}\right)^{4}}+\frac{12 B_{3} \lambda^{2}\left(\lambda^{3}+B_{3}^{2} \lambda\right)}{\left(\lambda^{2}-B_{4}^{2}\right)^{4}} \\
+\frac{12 B_{5} \lambda^{2}\left(\lambda^{3}+B_{5}^{2} \lambda\right)}{\left(\lambda^{2}-B_{6}^{2}\right)^{4}}
\end{array}\right],
\end{gathered}
$$

\section{B. MTDM TRANSMISSION BIT RATE MODEL ANALYSIS}

The maximum transmission bit rates are determined by numerous factors, including the signal modulation rate, the transmission bandwidth through the transmission media, and the response time of the optoelectronic devices. The total chromatic dispersion in single mode fiber that limits the transmission bit rates in system based UWWDM advanced optical communication network can be calculated as [13]:

$$
D_{t}=-\left(M_{m d}+M_{w d}\right), n \mathrm{sec} / n m \cdot k m
$$

Where $\mathrm{M}_{\mathrm{md}}$ is the material dispersion coefficient in $\mathrm{nsec} / \mathrm{nm} . \mathrm{km}, \mathrm{M}_{\mathrm{wd}}$ is the waveguide dispersion coefficient in $\mathrm{nsec} / \mathrm{nm} . \mathrm{km}$. The material dispersion coefficient is given as follows:

$$
M_{m d}=-\frac{\lambda_{s}}{c} \frac{d^{2} n}{d \lambda^{2}}-\frac{\Delta \lambda}{2 c}\left(\lambda \frac{d^{3} n}{d \lambda^{3}}+\frac{d^{2} n}{d \lambda^{2}}\right),
$$

The waveguide dispersion coefficient is given by:

$$
M_{w d}=-n_{2}\left(\frac{\Delta n}{c \lambda_{s}}\right) F(V),
$$

Where $\mathrm{n}_{2}$ is the refractive-index of the cladding material, $\Delta \mathrm{n}$ is the relative refractive-index difference, $\lambda_{\mathrm{s}}$ is the optical signal wavelength, $F(V)$ is a function of $\mathrm{V}$ number or normalized frequency. Based on the work [13], function F (V) is a function of $\mathrm{V}$ as:

$$
F(V)=1.38 V-6.98 V^{2}+13.45 V^{3}-4.84 V^{4}-1.48 V^{5} \text {, }
$$

The total pulse broadening, $\Delta \tau$, due to the effect of total chromatic dispersion is given as follows:

$$
\Delta \tau=D_{t} . \Delta \lambda . L, \quad n \sec
$$

Where $\Delta \lambda$ is the spectral linewidth of the used optical source in $\mathrm{nm}$, and $\mathrm{L}$ is the fiber cable length in $\mathrm{km}$. The MTDM bit rate per channel for silica core cable material is given as follows [14]:

$$
B_{r m c}=\frac{1}{4 \Delta \tau}=\frac{0.25}{\Delta \tau}, \text { Gbit } / \mathrm{sec} / \text { channel }
$$

Then the MTDM bit rate per link for silica core cable material is given by:

$$
B_{r m l}=\frac{0.25 * N_{\text {link }}}{\Delta \tau}, \quad \text { Gbit } / \mathrm{sec} / \text { link }
$$

\section{SOLITON TRANSMISSION BIT RATE MODEL ANALYSIS}

The term soliton has recently been coined to describe a pulse-like non-linear wave having unchanged shape and speed. The balance between the non-linearity effects from one side and the dispersion effects from the other side creates a solitary wave. The dispersion of a medium (in the absence of non-linearity) makes the various frequency components propagate at different velocities; while the non-linearity (in the absence of dispersion) causes the pulse energy to be continually injected, via harmonic generation, into higher frequency modes. The main types of soliton are the bright (light) soliton and the dark soliton (represented by absent pulse in alight wave). The total bandwidth is based on the total chromatic dispersion coefficient $D_{t}$ where:

$$
\mathrm{D}_{\mathrm{t}}=\mathrm{D}_{\mathrm{m}}+\mathrm{D}_{\mathrm{w}}
$$

Both $D_{m}$, and $D_{w}$ are given by (for the fundamental mode):

$$
D_{m}=-\frac{\lambda}{c}\left(\frac{d^{2} n}{d \lambda^{2}}\right), n \sec / n m . k m
$$




$$
D_{w}=-\left(\frac{n_{\text {cladding }}}{c n}\right)\left(\frac{\Delta n}{\lambda}\right) Y, n \mathrm{sec} / \mathrm{nm} \cdot \mathrm{km}
$$

Where $\mathrm{c}$ is the velocity of the light, $3 \times 10^{8}$ $\mathrm{m} / \mathrm{sec}, \mathrm{n}$ is the refractive-index of the fiber cable core, $\mathrm{Y}$ is a function of wavelength, the relative refractive-index difference $\Delta \mathrm{n}$ is given by:

$$
\Delta n=\frac{n-n_{\text {cladding }}}{n},
$$

In any infinitesimal segment of fiber, dispersion on one hand and non linearity of the refractive-index on the other hand produce infinitesimal modulation angles which exactly compensate reciprocally. Under such conditions the pulse shape is the same everywhere. All this provided that a soliton waveform be used with a peak power [15]:

$$
P_{1}=\frac{\Delta \lambda^{3} D_{t} A_{e f f}}{4 \pi^{2} c n_{2} t_{0}^{2}},
$$

Where $\mathrm{n}_{2}$ is the nonlinear Kerr coefficient, $2.6 \mathrm{x}$ $10^{-20} \mathrm{~m}^{2} /$ Watt, $\Delta \lambda$ is the spectral line width of the optical source in $\mathrm{nm}, \mathrm{P}_{1}$ is the peak power in watt, $A_{\text {eff }}$ is the effective area of the cable core fiber in $\mu \mathrm{m}^{2}, \mathrm{D}_{\mathrm{t}}$ is the total chromatic dispersion coefficient in $\mathrm{nsec} / \mathrm{nm} . \mathrm{km}$. Then the pulse intensity width in nsec is given by:

$$
t_{0}=\sqrt{\frac{\Delta \lambda^{3} D_{t} A_{e f f}}{4 \pi^{2} P_{1} n_{2} c}}, \quad n \mathrm{sec}
$$

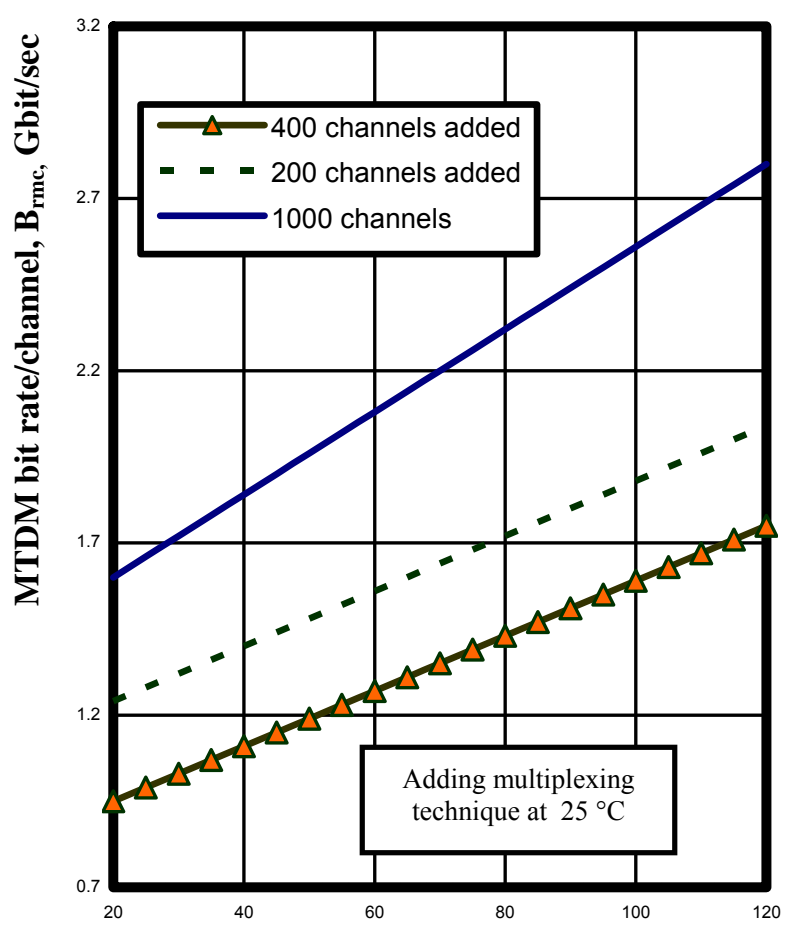

Number of links in the fiber cable core, $\mathrm{N}_{\mathrm{L}}$

Fig. 4 - Variations of MTDM bit rate/channel with number of links in fiber cable core at assumed set of parameters

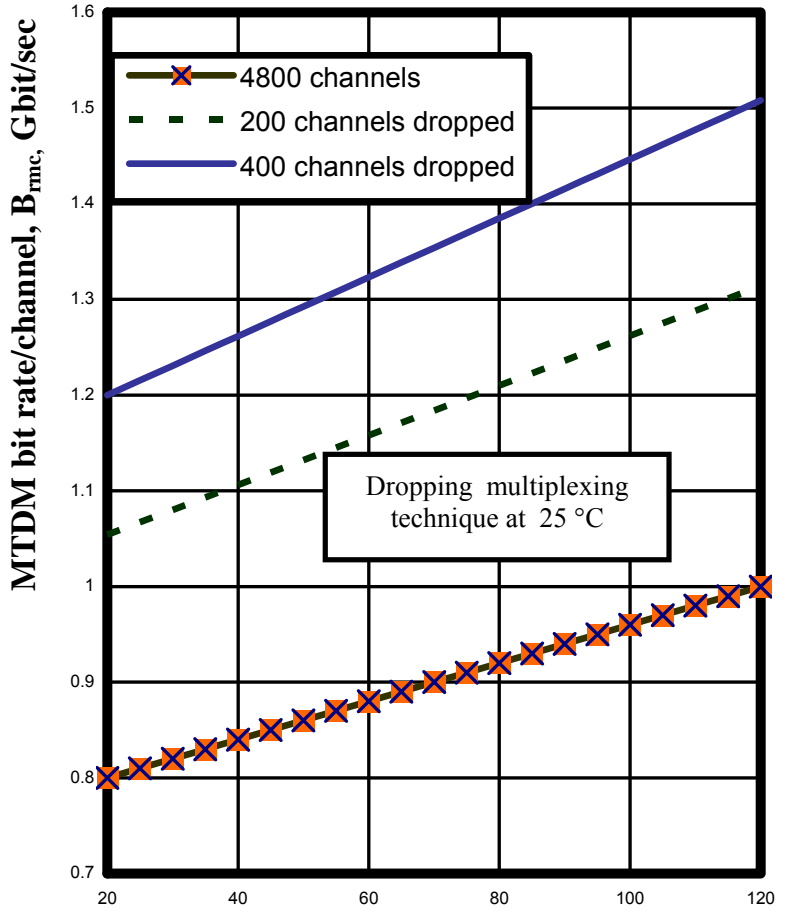

Number of links in the fiber cable core, $\mathbf{N}_{L}$

Fig. 5 - Variations of MTDM bit rate/channel with number of links in fiber cable core at assumed set of parameters

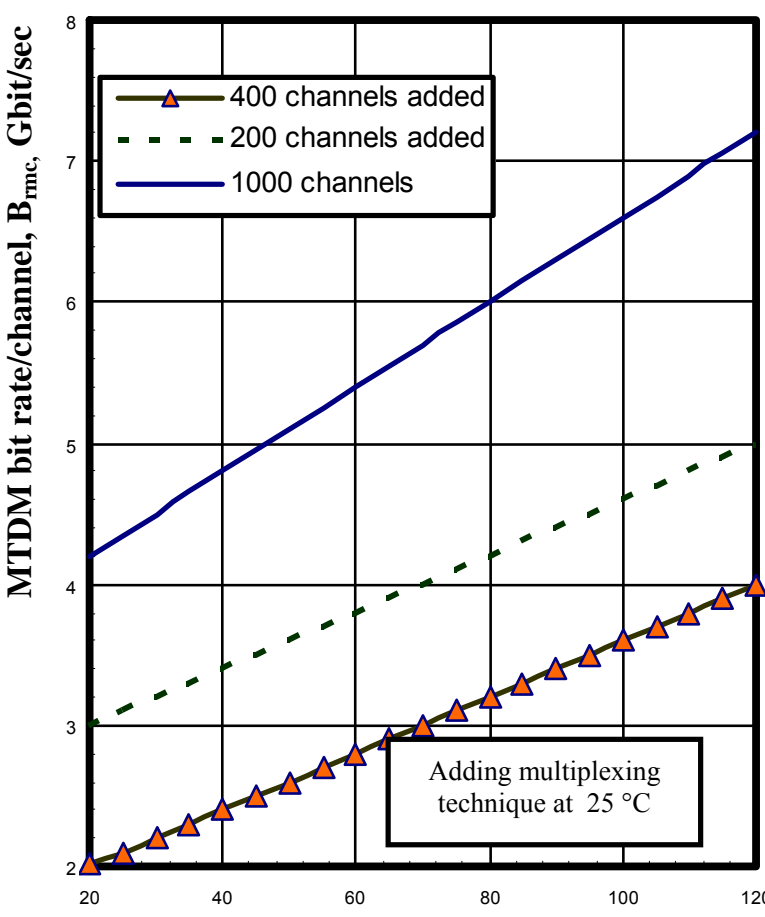

Number of links in the fiber cable core, $\mathrm{N}_{L}$

Fig. 6 - Variations of MTDM bit rate/link with number of links in fiber cable core at assumed set of parameters 


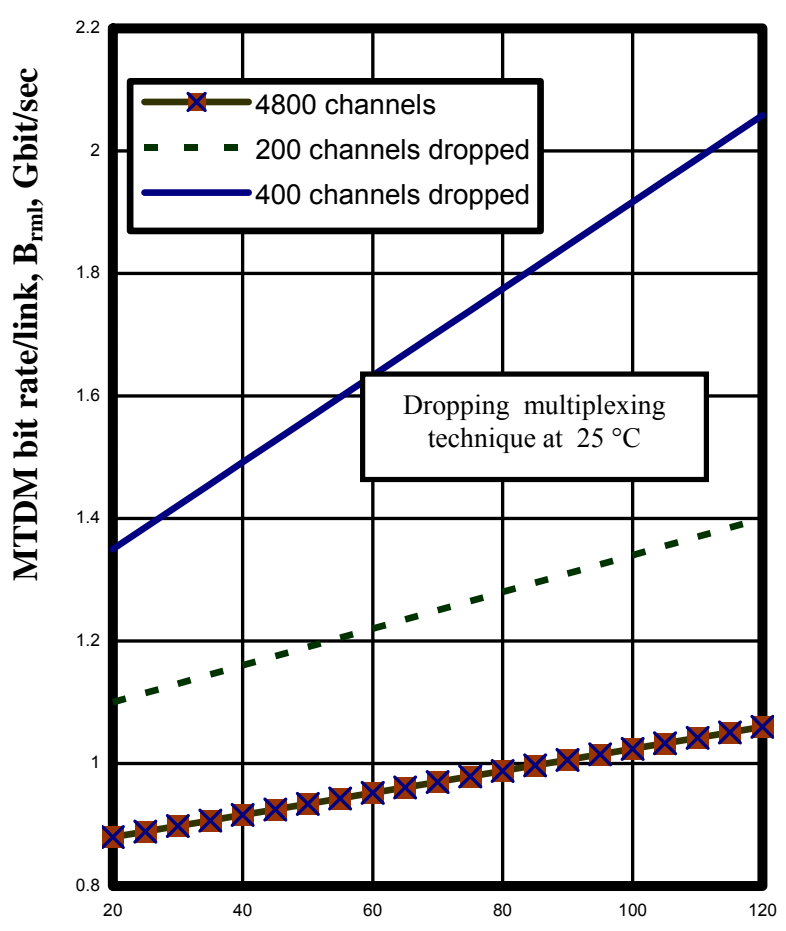

Number of links in the fiber cable core, $\mathrm{N}_{\mathrm{L}}$

Fig. 7 - Variations of MTDM bit rate/link with number of links in fiber cable core at assumed set of parameters

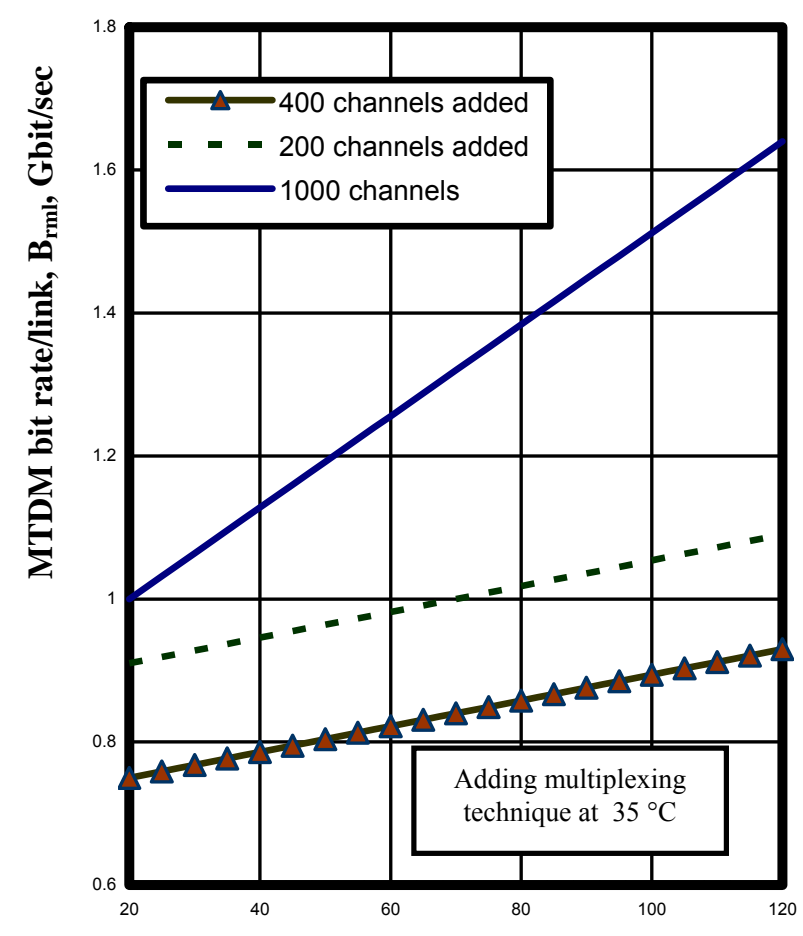

Number of links in the fiber cable core, $N_{L}$

Fig. 8 - Variations of MTDM bit rate/channel with number of links in fiber cable core at assumed set of parameters

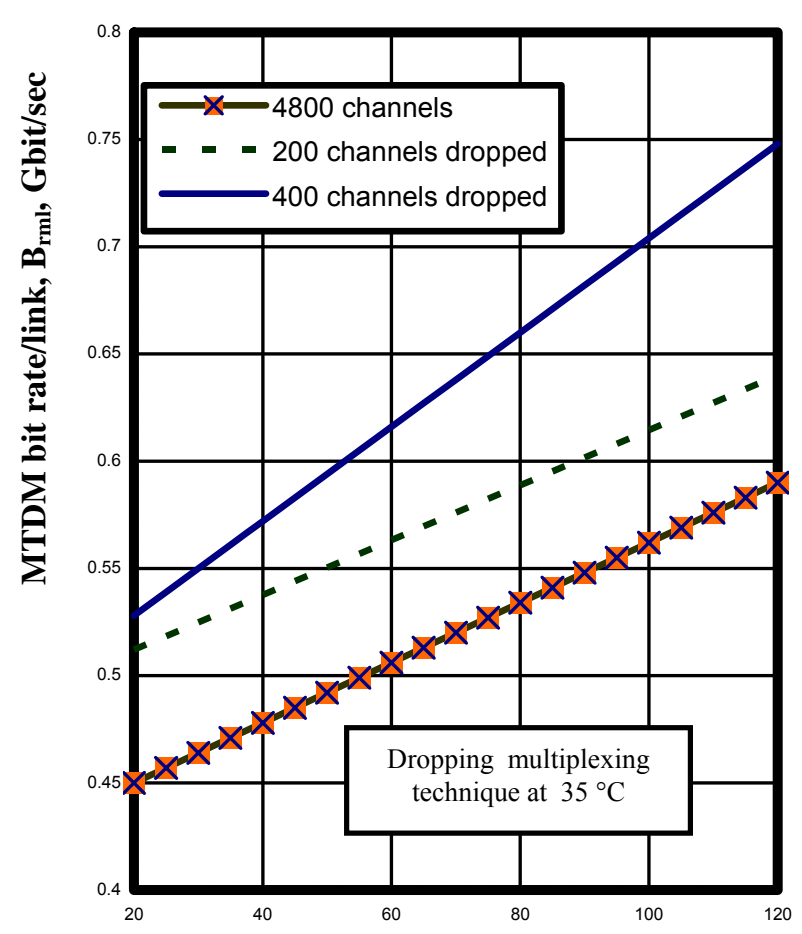

Number of links in the fiber cable core, $\mathrm{N}_{\mathrm{L}}$

Fig. 9 - Variations of MTDM bit rate/channel with number of links in fiber cable core at assumed set of parameters

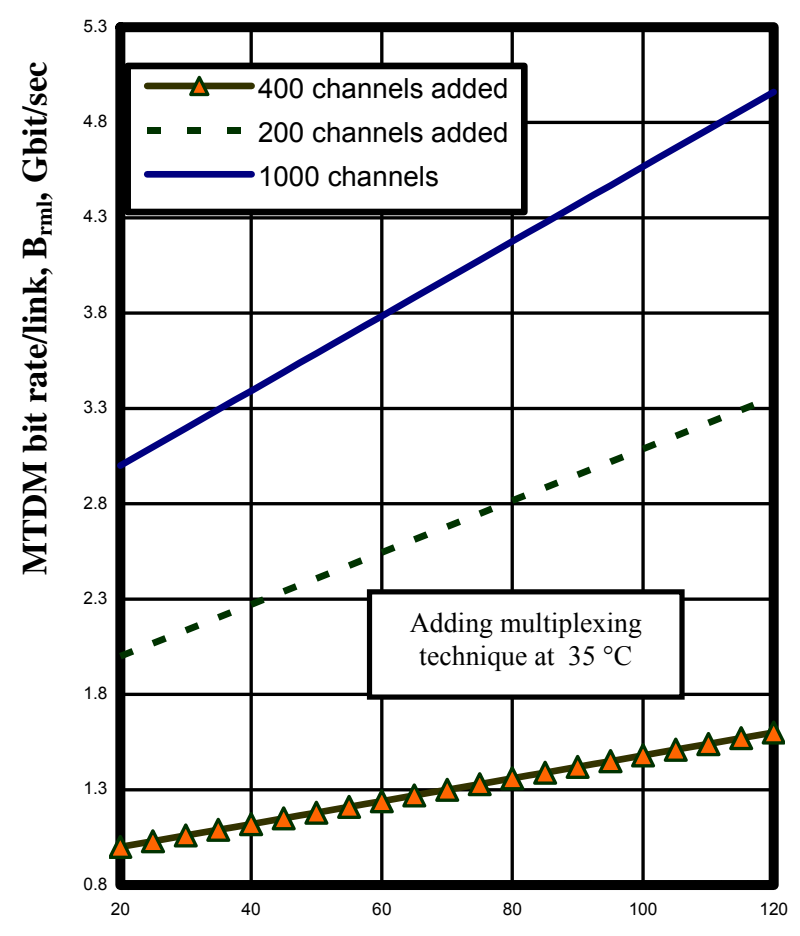

Number of links in the fiber cable core, $\mathbf{N}_{\mathrm{L}}$

Fig. 10 - Variations of MTDM bit rate/link with number of links in fiber cable core at assumed set of parameters 


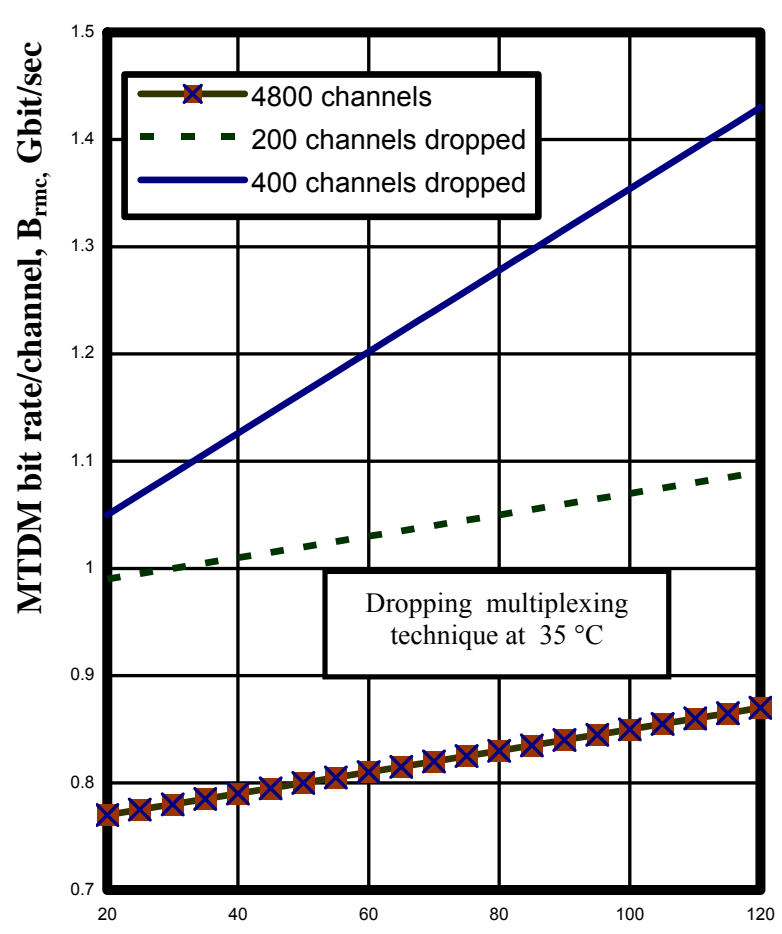

Number of links in the fiber cable core, $\mathrm{N}_{\mathrm{L}}$

Fig. 11 - Variations of MTDM bit rate/link with number of links in fiber cable core at assumed set of parameters

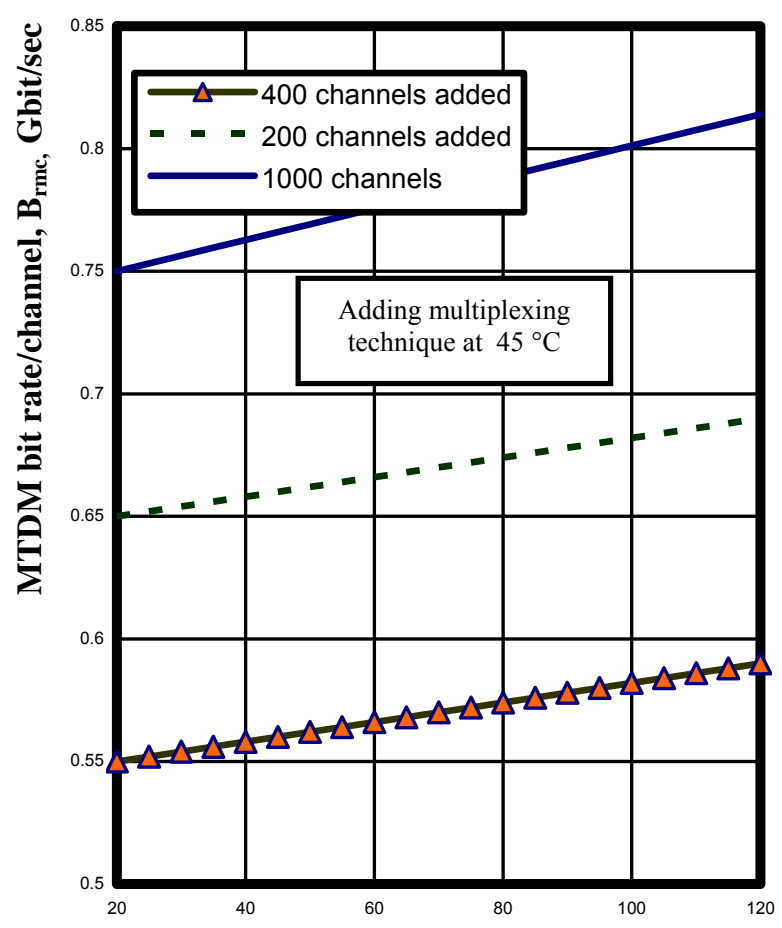

Number of links in the fiber cable core, $\mathrm{N}_{\mathrm{L}}$

Fig. 12 - Variations of MTDM bit rate/channel with number of links in fiber cable core at assumed set of parameters

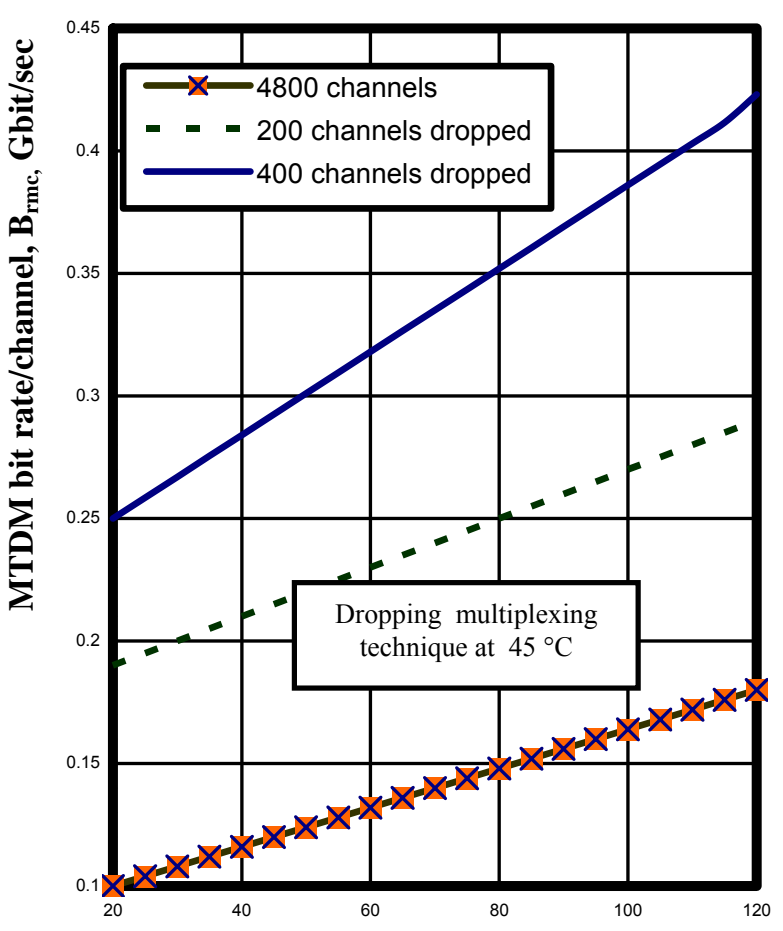

Number of links in the fiber cable core, $\mathrm{N}_{\mathrm{L}}$

Fig. 13 - Variations of MTDM bit rate/channel with number of links in fiber cable core at assumed set of parameters

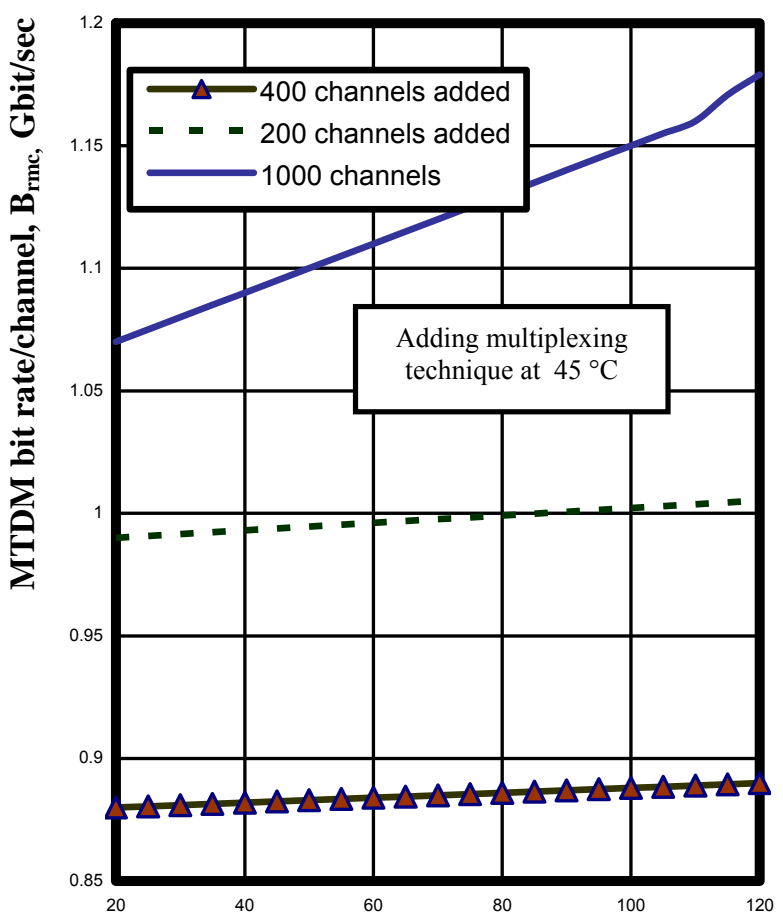

Number of links in the fiber cable core, $\mathbf{N}_{\mathrm{L}}$

Fig. 14 - Variations of MTDM bit rate/link with number of links in fiber cable core at assumed set of parameters 


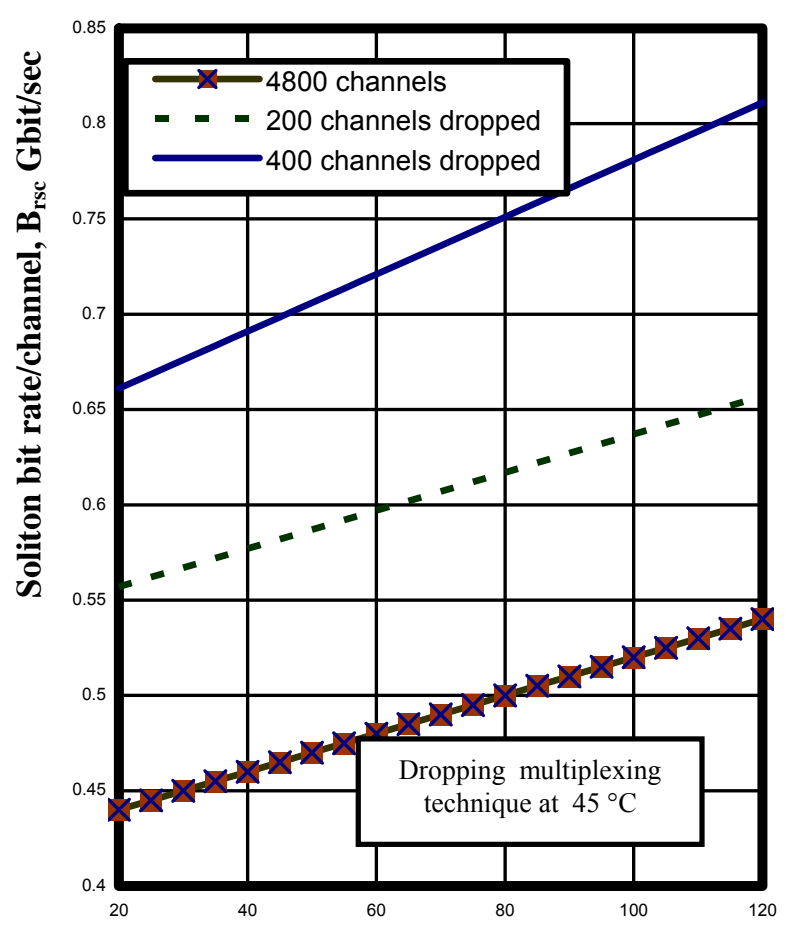

Number of links in the fiber cable core, $\mathrm{N}_{\mathrm{L}}$

Fig. 15 - Variations of MTDM bit rate/link with number of links in fiber cable core at assumed set of parameters

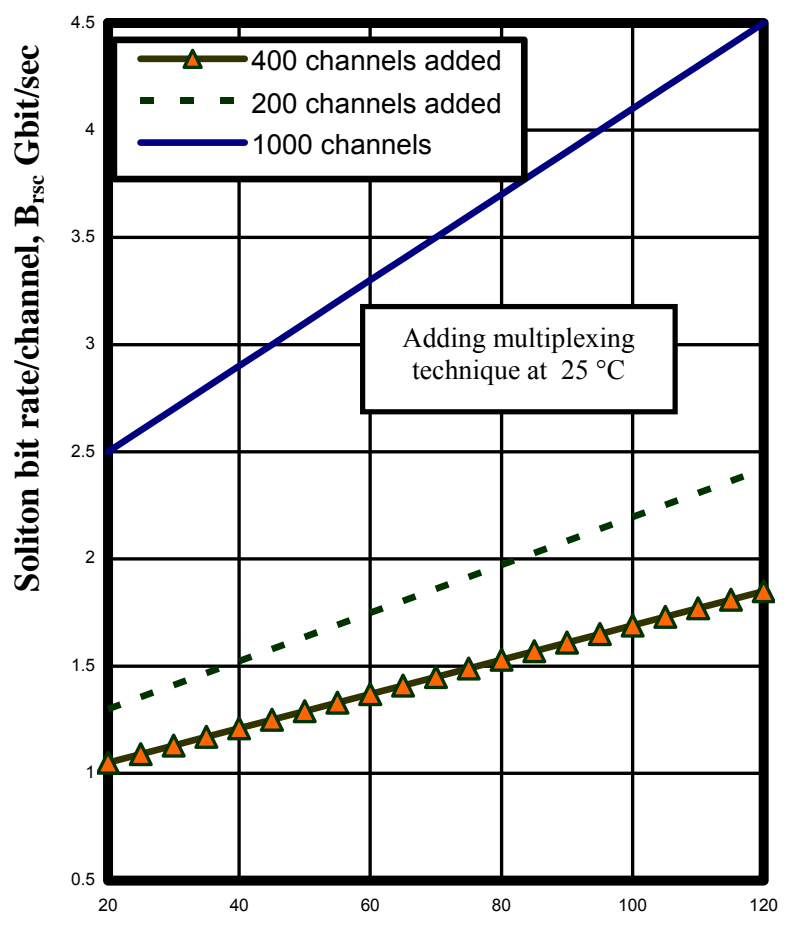

Number of links in the fiber cable core, $N_{L}$

Fig. 16 - Variations of soliton bit rate/channel with number of links in fiber cable core at assumed set of parameters

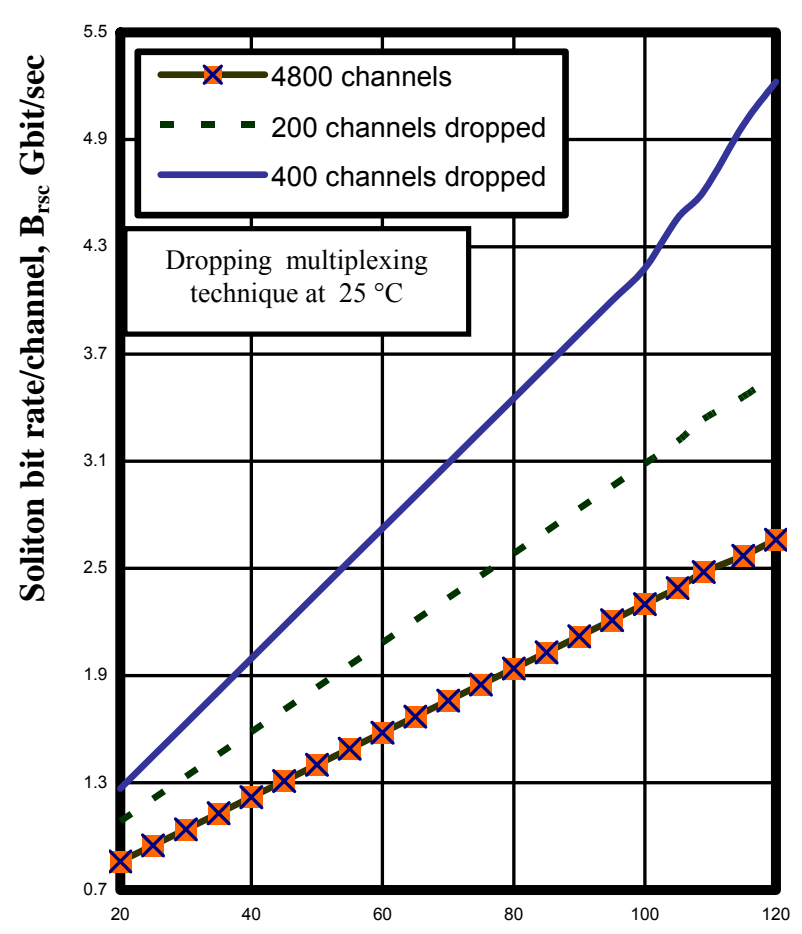

Number of links in the fiber cable core, $\mathrm{N}_{\mathrm{L}}$

Fig. 17 - Variations of soliton bit rate/channel with number of links in fiber cable core at assumed set of parameters

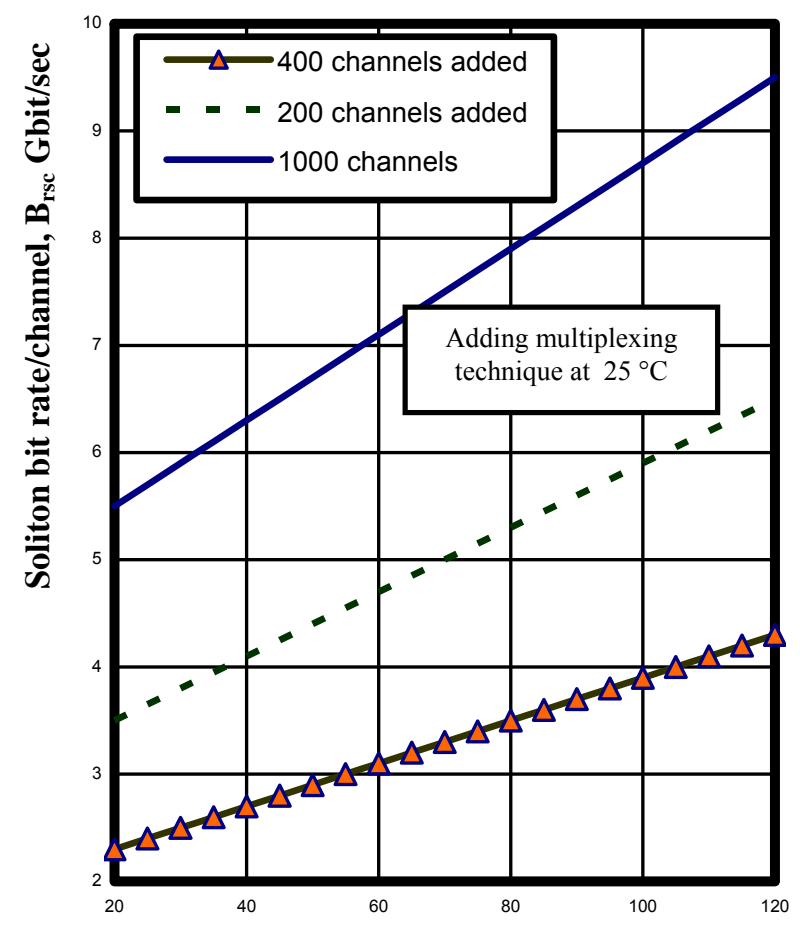

Number of links in the fiber cable core, $N_{L}$

Fig. 18 - Variations of soliton bit rate/link with number of links in fiber cable core at assumed set of parameters 


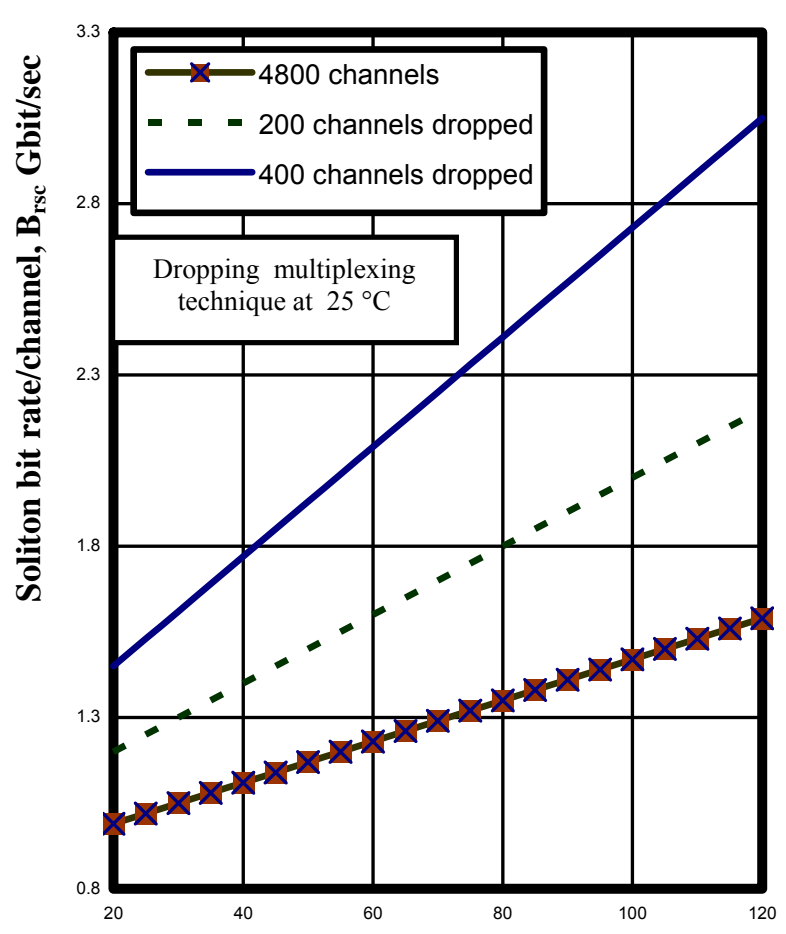

Number of links in the fiber cable core, $\mathrm{N}_{\mathrm{L}}$

Fig. 19 - Variations of soliton bit rate/link with number of links in fiber cable core at assumed set of parameters

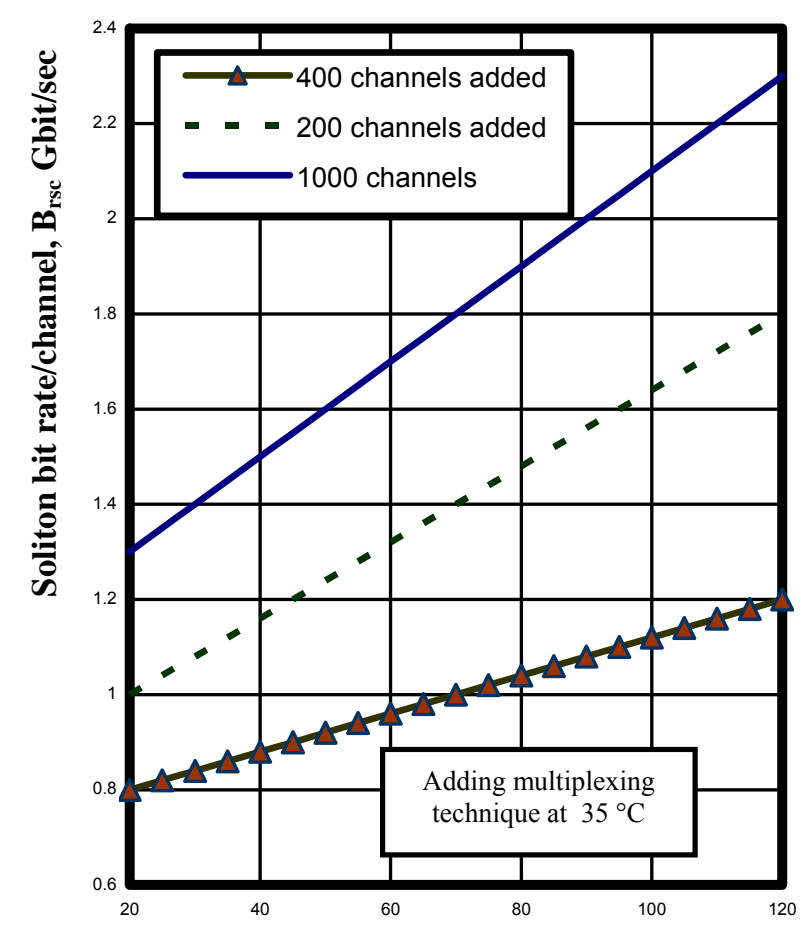

Number of links in the fiber cable core, $\mathrm{N}_{\mathrm{L}}$

Fig. 20 - Variations of soliton bit rate/channel with number of links in fiber cable core at assumed set of parameters

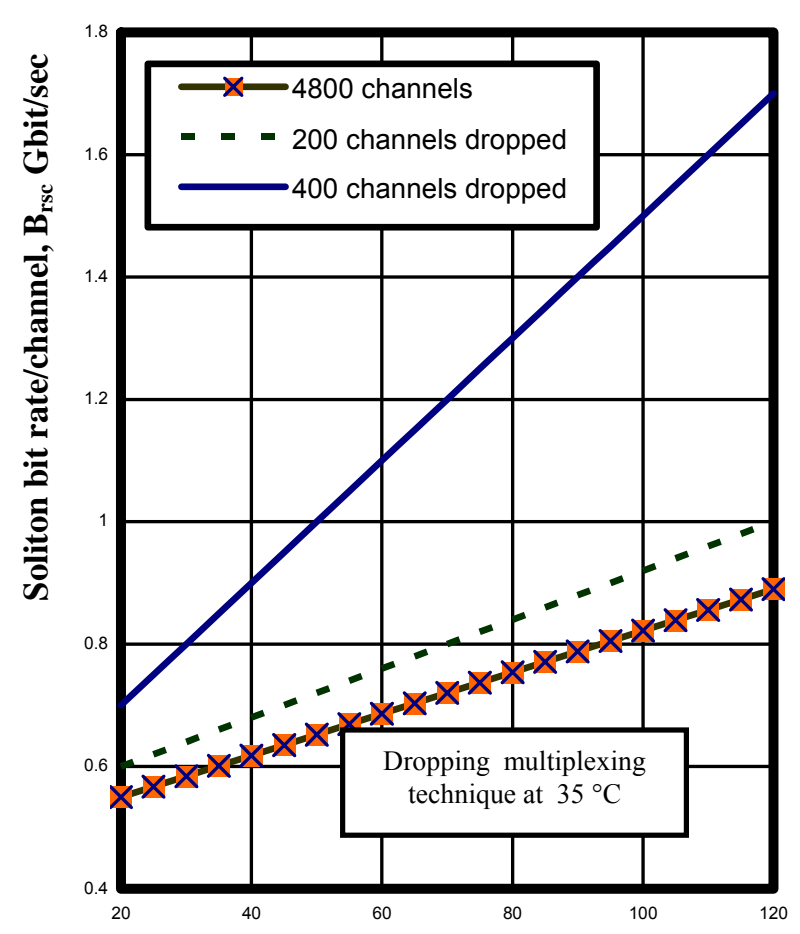

Number of links in the fiber cable core, $\mathrm{N}_{\mathrm{L}}$

Fig. 21 - Variations of soliton bit rate/channel with number of links in fiber cable core at assumed set of parameters

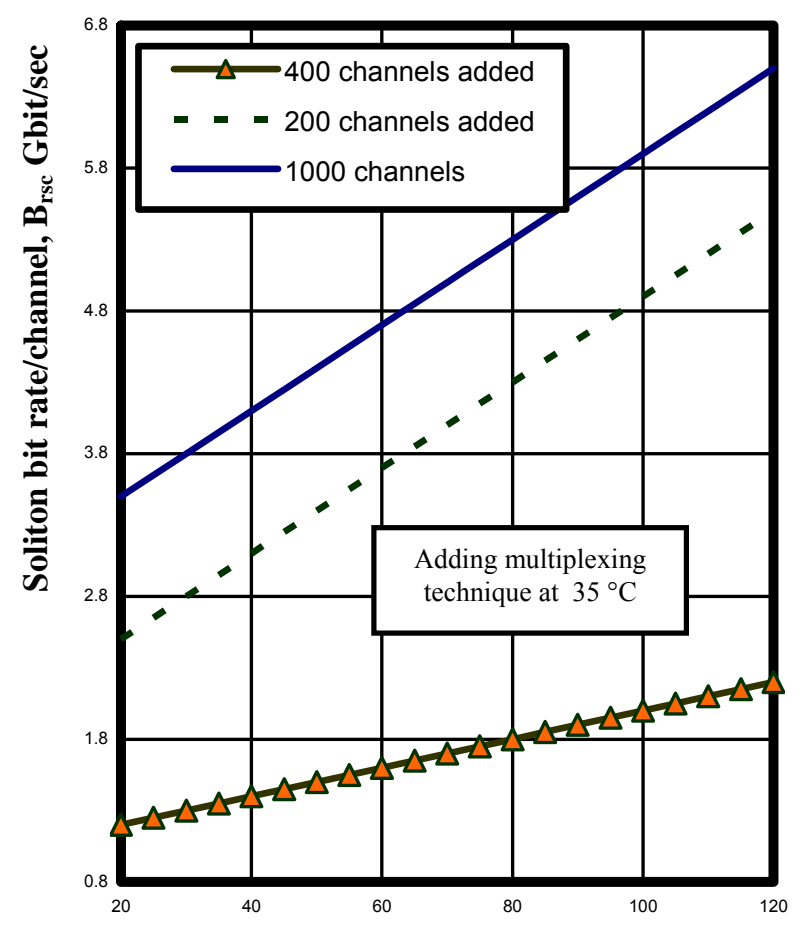

Number of links in the fiber cable core, $\mathbf{N}_{\mathrm{L}}$

Fig. 22 - Variations of soliton bit rate/link with number of links in fiber cable core at assumed set of parameters 


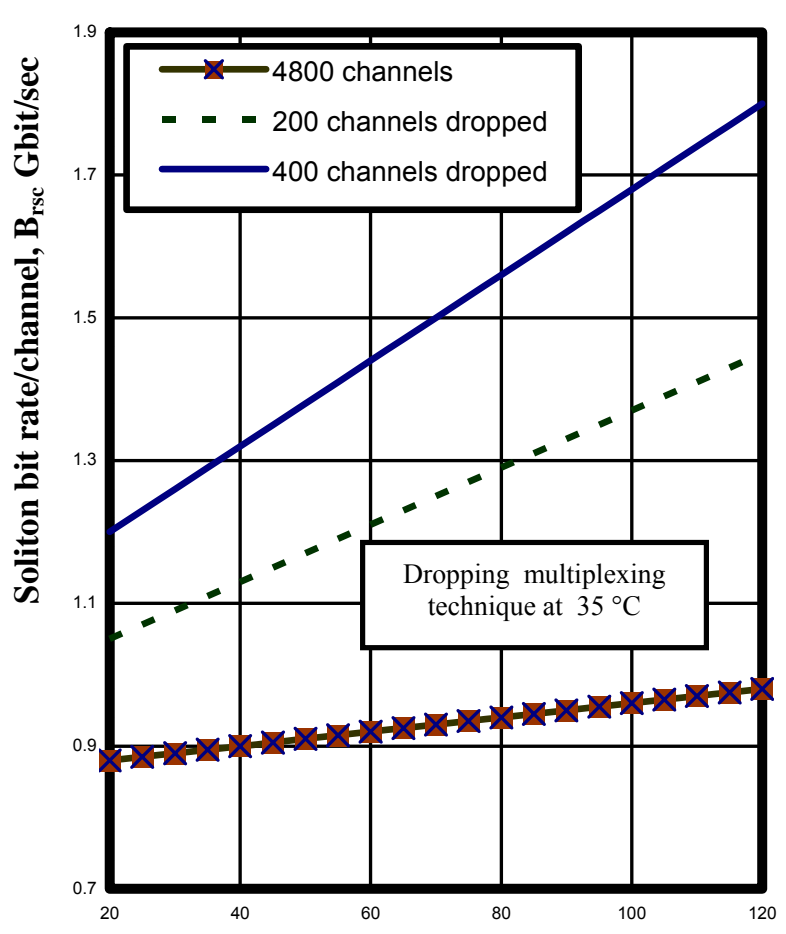

Number of links in the fiber cable core, $\mathrm{N}_{\mathrm{L}}$

Fig. 23 - Variations of soliton bit rate/link with number of links in fiber cable core at assumed set of parameters

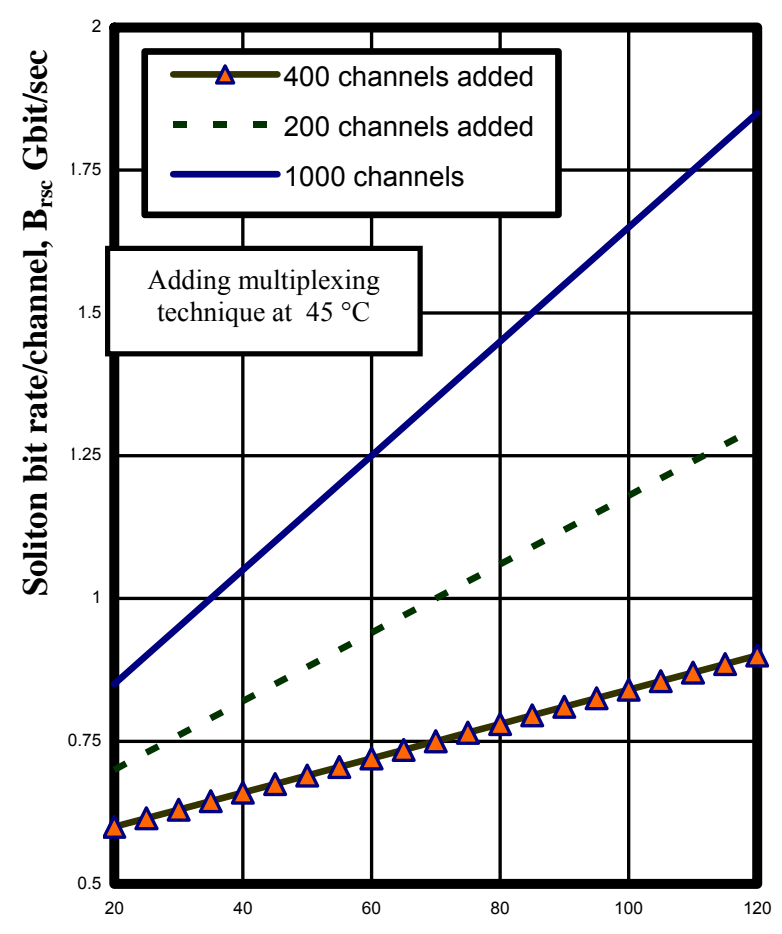

Number of links in the fiber cable core, $\mathrm{N}_{\mathrm{L}}$

Fig. 24 - Variations of soliton bit rate/channel with number of links in fiber cable core at assumed set of parameters

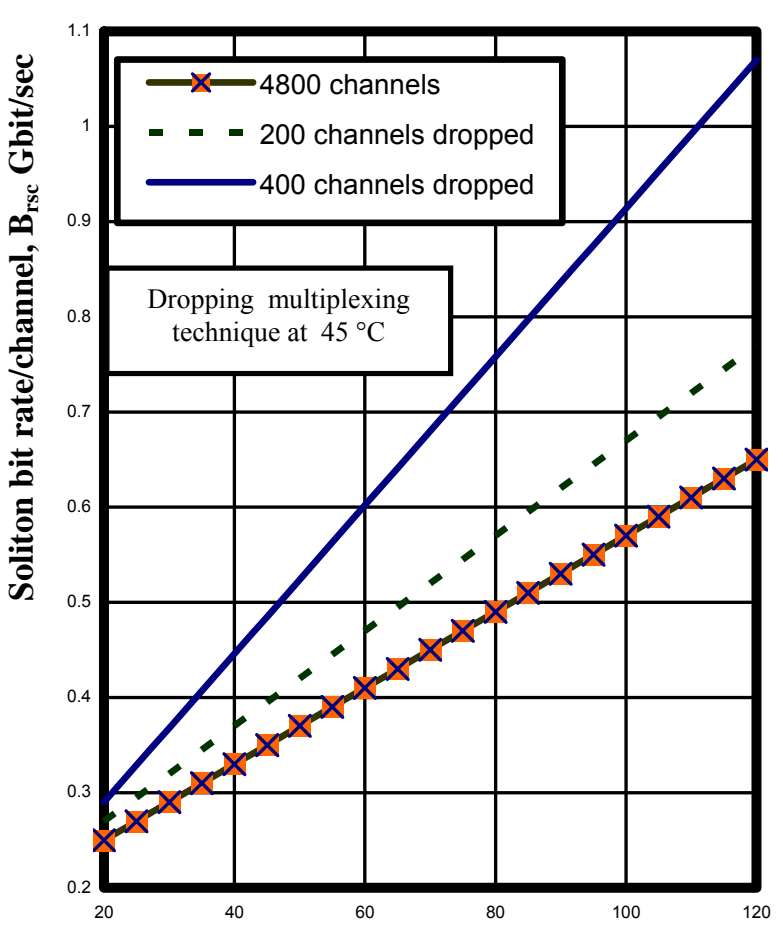

Number of links in the fiber cable core, $\mathbf{N}_{\mathrm{L}}$

Fig. 25 - Variations of soliton bit rate/channel with number of links in fiber cable core at assumed set of parameters

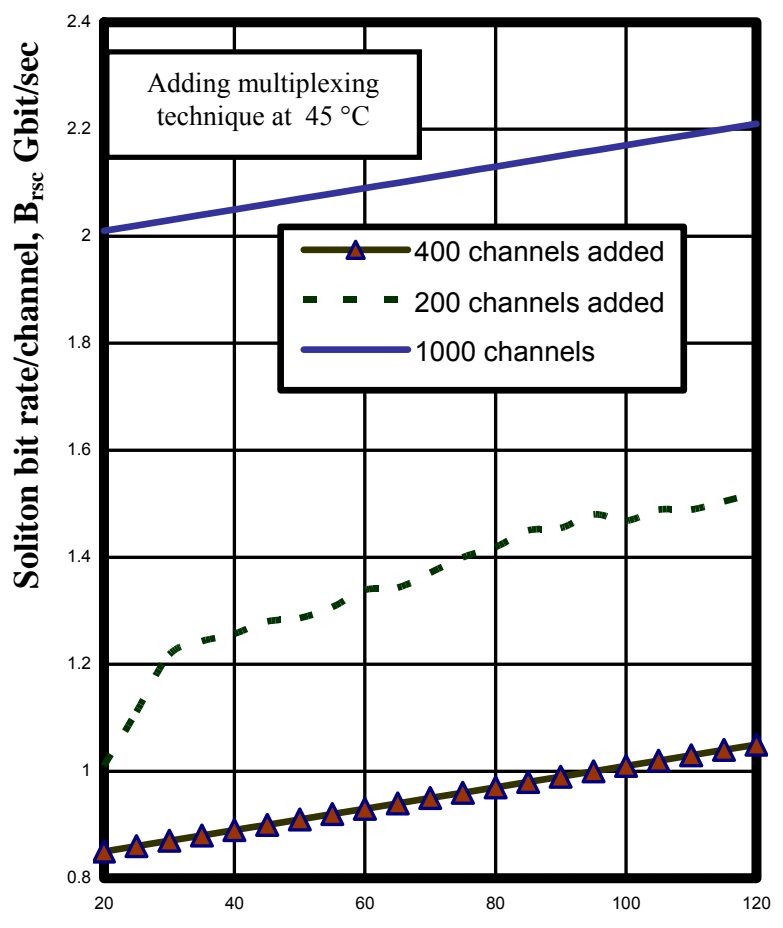

Number of links in the fiber cable core, $\mathbf{N}_{\mathrm{L}}$

Fig. 26 - Variations of soliton bit rate/link with number of links in fiber cable core at assumed set of parameters 


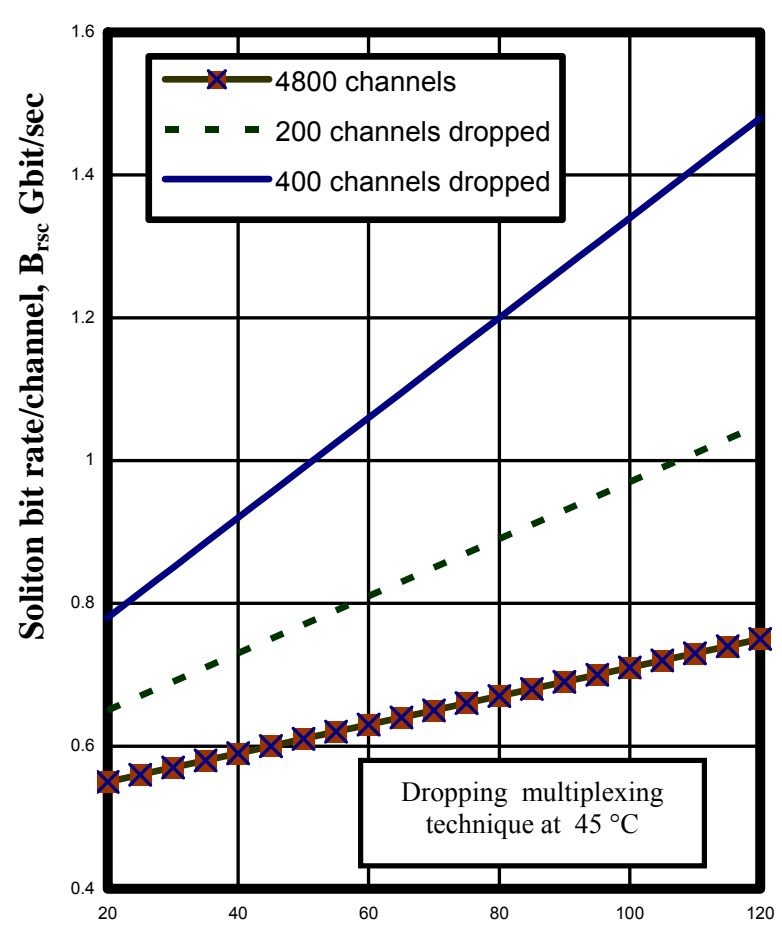

Number of links in the fiber cable core, $\mathrm{N}_{\mathrm{L}}$

Fig. 27 - Variations of soliton bit rate/link with number of links in fiber cable core at assumed set of parameters

Then the Soliton transmission bit rate per optical network channel or unit is given as follows:

$$
B_{r s c}=\frac{1}{10 t_{0}}=\frac{0.1}{t_{0}}, \text { Gbit } / \mathrm{sec} / \text { channel }
$$

Then the Soliton transmission bit rate per link is:

$$
B_{r s l}=\frac{0.1 * N_{\text {link }}}{t_{0}}, \quad \text { Gbit } / \mathrm{sec} / \text { link }
$$

\section{RESULTS AND DISCUSSIONS}

We have investigated optical add drop multiplexers with different multiplexing techniques in advanced optical communication and photonic networks for upgrading the facility of add or drop optical channels along the optical networks in the interval of $1.45 \mu \mathrm{m}$ to $1.65 \mu \mathrm{m}$ under the set of affecting and controlling parameters at temperature range varies from $25^{\circ} \mathrm{C}$ to $45^{\circ} \mathrm{C}$. The following set of the numerical data of our simulation system model design are employed to obtain different propagation or transmission bit rates either per link or per channel in the case of add or drop optical channels as follows: $1.45 \leq \lambda_{\text {si }}$, optical signal wavelength, $\mu \mathrm{m} \leq 1.65,2 \leq \mathrm{P}_{\mathrm{s}}$, optical signal power, mwatt $\leq 20$, refractive-index of the cladding material, $\mathrm{n}_{2}=1.445, \mathrm{~N}_{\mathrm{L}}$ : total number of links up to 120 links, spectral linewidth of the optical source, $\mathrm{A}_{\text {efff }}=85 \mu \mathrm{m}^{2}, \Delta \lambda_{\mathrm{s}}=0.1 \mathrm{~nm}$, relative refractive-index difference, $\Delta \mathrm{n}=0.007, \mathrm{~N}_{\mathrm{t}}$ : total number of channels up to 4800 channels, $L$ is the fiber cable core length $=10 \mathrm{~km}$.

Based on the set of Figs (4-27), the following features are assured to present the importance role of OADMs in advanced optical communication networks within MTDM and soliton transmission techniques, UW-WDM, and UW-SDM multiplexing techniques as the following:

1) Figs. (4-7) have indicated that as the number of links in the fiber cable core increases, the MTDM bit rate either per link or per channel increase at the same number of transmitted, added or dropped channels. But as the number of added channels increase, the MTDM bit rate either per link or per channel decreases at the same number of links in the fiber cable core. Moreover, we can conclude that as the number of dropped channels increase, the MTDM transmission technique either per link or per channel increase at the same number of links in the fiber cable core at the constant value of room temperature.

2) Figs. (8-11) have demonstrated that as the number of links in the fiber cable core increases, the MTDM bit rate either per link or per channel increase at the same number of transmitted, added or dropped channels. But as the number of added channels increase, the MTDM bit rate either per link or per channel decreases at the same number of links in the fiber cable core. Also, we can observe that as the number of dropped channels increase, the MTDM transmission technique either per link or per channel increase at the same number of links in the fiber cable core at the constant value of temperature at $35^{\circ} \mathrm{C}$.

3) Figs. (12-15) have assured that as the number of links in the fiber cable core increases, the MTDM bit rate either per link or per channel increase at the same number of transmitted, added or dropped channels. But as the number of added channels increase, the MTDM bit rate either per link or per channel decreases at the same number of links in the fiber cable core. Therefore, we can conclude that as the number of dropped channels increase, the MTDM transmission technique either per link or per channel increase at the same number of links in the fiber cable core at the constant value of temperature at $45^{\circ} \mathrm{C}$.

4) Figs. (16-19) have indicated that as the number of links in the fiber cable core increases, the soliton bit rate either per link or per channel increase at the same number of transmitted, added or dropped channels. But as the number of added channels increase, the soliton bit rate either per link or per channel decreases at the same number of links in the fiber cable core. Moreover, we can conclude that as the number of dropped channels increase, the soliton transmission technique either 
per link or per channel increase at the same number of links in the fiber cable core at the constant value of room temperature.

5) Figs. (20-23) have demonstrated that as the number of links in the fiber cable core increases, the soliton bit rate either per link or per channel increase at the same number of transmitted, added or dropped channels. But as the number of added channels increase, the soliton bit rate either per link or per channel decreases at the same number of links in the fiber cable core. Also, we can observe that as the number of dropped channels increase, the soliton transmission technique either per link or per channel increase at the same number of links in the fiber cable core at the constant value of temperature at 35 ${ }^{\circ} \mathrm{C}$.

6) Figs. (24-27) have assured that as the number of links in the fiber cable core increases, the soliton bit rate either per link or per channel increase at the same number of transmitted, added or dropped channels. But as the number of added channels increase, the soliton bit rate either per link or per channel decreases at the same number of links in the fiber cable core. Therefore, we can conclude that as the number of dropped channels increase, the soliton transmission technique either per link or per channel increase at the same number of links in the fiber cable core at the constant value of temperature at $45^{\circ} \mathrm{C}$.

7) Figs. (4-27) have demonstrated that the MTDM and soliton bit rates either per channel or per link decrease as the ambient temperature of fiber cable core material increases for both adding or dropping transmitted channels multiplexing technique. Also, we have indicated that in the case of optical adding multiplexing technique, the increased number of transmitted channels and then the increased number of subscribers over optical communication network. Moreover, we have assured that in the case of optical dropping multiplexing technique, the increased MTDM and soliton bit rate either per link or per channel over optical communication network.

\section{CONCLUSIONS}

In a summary, we have presented analytically the importance role of OADMs in advanced optical communication networks over wide range of the affecting parameters. Also the investigation of MTDM and soliton transmission techniques are processed to handle both bit rates either per link or per channel and maximum number of transmitted channels for multi-links of fiber cable core. It is evident that the increased of number of links in the fiber cable core, the increased MTDM and soliton bit rates either per link or per channel for both adding or dropping channels multiplexing technique. Moreover, we have observed and demonstrated the increased of ambient temperature of fiber cable core, the decreased MTDM and soliton bit rates either per link or per channel for both adding or dropping transmitted channels multiplexing technique. We have demonstrated that the increased number of transmitted channels can be achieved within optical adding multiplexing technique and then to increase number of subscribers over optical communication network. Also, we have assured that the increased bit rate is achieved within optical dropping multiplexing technique. Therefore, we have presented the high performance and important role of OADMs to allow subscribers to optimize the use of existing fiber cable by adding or dropping transmitted channels and then maximize fiber cable bandwidth.

\section{REFERENCES}

[1] St. Fischer, M. Dulk, E. Gamper, W. Vogt, W. Hunziker, E. Gini, A. Buxens, and A. Clausen. All-optical regenerative OTDM add-drop multiplexing at $40 \mathrm{Gbit} / \mathrm{sec}$ using monolithic InP Mach-Zehnder interferometer, IEEE Photonics Technology Letters, 2000, Vol. 12, No. 3, pp. 335-337.

[2] B. Olsson, P. Ohlen, L. Rau and D. Blumenthal. A simple and robust $40-\mathrm{Gb} / \mathrm{s}$ wavelength converter using fiber cross-phase modulation and optical filtering, IEEE Photonics Technology Letters, 2000, Vol. 12, No. 7, pp. 846-848.

[3] K. Iwatsuki, J. Kani and H. Suzuki. Access and metro optical networks based on WDM technologies, J. Lightwave Technol., 2004, Vol. 22, No. 15, pp. 2623-2630.

[4] J. Castro, D. Geraghty, B. West and S. Honkanen. Fabrication and comprehensive modeling of ion-exchanged bragg optical add drop multiplexers, Appl. Opt., 2004, Vol. 43, No. 5, pp. 6166-6173.

[5] J. Castro, D. Geragthy, S. Honkanen, C. Greiner, D. Iazikov and T. Mossberg. Demonstration of mode conversion using antisymmetric waveguide Bragg gratings, Opt. Express, 2005, Vol. 13, No. 2, pp. 4180-4184.

[6] C. Greiner, T. Mossberg and D. Iazikov. Bandpass engineering of lithographically scribed channel-waveguide Bragg gratings, Opt. Lett., 2004, Vol. 29, No. 4, pp. 806-808.

[7] M. Aslund, J. Canning, L. Poladian, C. Sterke and A. Judge. Antisymmetric grating coupler: experimental results, Appl. Opt., 2003, Vol. 42, No. 2, pp. 6578-6583. 
[8] C. Fouli and M. Maier. OCDMA and optical coding principles, applications, and challenges, IEEE Comm. Mag., 2007Vol. 45, No. 8, pp. 2734.

[9] H. Hashimoto, A. Ueno, M. Taniue, S. Kawase, O. Koyama and Y. Katsuyama. Design and Control System over WWW for Regional CWDM Optical IP Networks with Optical Add/Drop Multiplexers, International Journal of Innovative Computing, Information and Control, 2008, Vol. 4, No. 6, pp. 1299-1313.

[10] R. A. Chraplyvy. Limitation on lightwave communication systems imposed by optical fiber nonlinearities, J. Lightwave Technol., 1990, Vol. 18, No.10, pp. 1548-1557.

[11] T. Otani, T. Miyazaki, H. S. Carassa and S. Yamamot. $40-\mathrm{Gb} / \mathrm{sec}$ optical 3R regenerator using electro absorption modulators for optical communication networks, J. Lightwave Technol., 2002, Vol. 20, No. 2, pp. 195-200.

[12] W. Fleming. Dispersion in $\mathrm{GeO}_{2}-\mathrm{SiO}_{2}$ glasses, Applied Optics, 1985, Vol. 23, No. 24, pp. 4486-4493.

[13] R. J. S. Bates and K. P. Jackson. Improved multimode fiber link BER calculations due to modal noise and non self-pulsating laser diodes, Optic, Quantum Electron., 1995, Vol. 27, No. 13 , pp. 203-224.

[14] A. A. M. El-Naser, A. S. El-Fattah and Z. N. A. Rashed. High channel arrayed waveguide grating (AWG) in wavelength division multiplexing passive optical networks (WDM-PONs), IJCSNS International Journal of Computer Science and Network Security, 2009, Vol. 9, No. 1, pp. 253-259.

[15] G. Yabre. Theoretical Investigation on the Dispersion of Graded-Index Polymer Optical Fiber, Journal of Lightw. Technol., 2000, Vol. 18 , No. 16 , pp. 869-882.

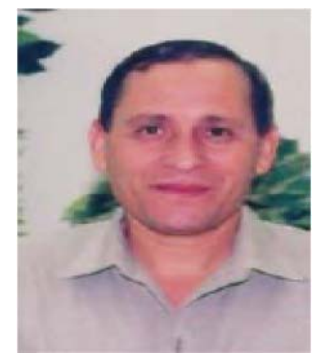

Abd-Elnaser A. Mohammed, received $P$ h.D scientific degree from the faculty of Electronic Engineering, Menoufia University in 1994. Now, his job career is Assoc. Prof. Dr. in Electronics and Electrical Communication Engineering department. Currently, his field, and research interest in the all passive optical and communication Networks, analog-digital communication systems, optical systems, and advanced optical comnmunication networks.

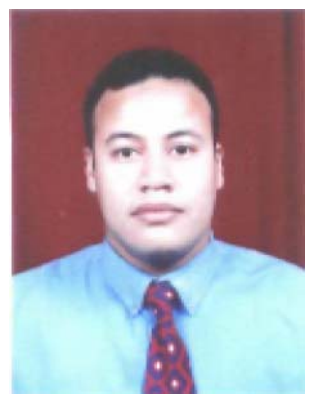

Ahmed Nabih Zaki Rashed was, born in Menouf, Menoufia State. Egypt, in 1976. Received the B.Sc. and M.Sc. practical scientific degrees in the Electronics and Electrical Communication Engineering Department from Faculty of Electronic Engineering, Menoufia University in 1999 and 2005, respectively. Curreuntly, his field interest and working toward the Ph.D degree in Active and Passive Optical Networks (PONs). His theoretical and practical scientific research mainly focuses on the transmission data rates and distance of optical access networks.

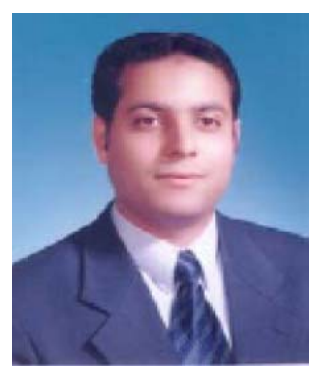

Mohamoud M. A. Eid was born in Gharbiya State, Egypt, in 1977. Received the B.SC. and $M$. Sc. degrees in the Electronics Communication Engineering Department from Faculty of Electronic Engineering, Menoufia University in 2002 and 2007. Currently, his working toward the Ph.D degree in Ultra wide wavelength division multiplexing. 